\title{
Effectiveness of Price Limit on Stock Market Network: A Time-Migrated DCCA Approach
}

\author{
Hongzeng He $\mathbb{C}^{1,2}$ and Shufen Dai $\mathbb{i D}^{1}$ \\ ${ }^{1}$ School of Economics and Management, University of Science and Technology Beijing, Beijing 100083, China \\ ${ }^{2}$ Returned Overseas Talent and Expert Service Center, MOHRSS, Beijing 100083, China \\ Correspondence should be addressed to Hongzeng He; hehongzeng@126.com
}

Received 5 July 2020; Accepted 21 July 2020; Published 18 January 2021

Guest Editor: Xiaodi Li

Copyright (c) 2021 Hongzeng He and Shufen Dai. This is an open access article distributed under the Creative Commons Attribution License, which permits unrestricted use, distribution, and reproduction in any medium, provided the original work is properly cited.

\begin{abstract}
In this paper, we investigated the effectiveness of price limit on stock market with the correlation study and complex network technology. We proposed a time-migrated DCCA cross-correlation coefficient which is beneficial to detect the asynchronous correlations of nonstationary time series. The stock market network is constructed with the threshold method based on timemigrated DCCA. The effectiveness of the price limit during the stock market crash period is studied based on the time-migrated DCCA stock market network. The results indicate that the time-migrated DCCA ensures more relevant results than the equal-time DCCA method. An interesting finding is that the price limit has different effects on the stock market network at different stages of dynamic evolution. Market stabilization will be lowered and the systemic risk will be increased if the price limit is enhanced. Such studies are relevant for a better understanding of the stock market and have a significant contribution to the stock market in reality.
\end{abstract}

\section{Introduction}

It is believed that a number of systems could be described by complex networks, including traffic systems, ecological systems, and financial systems. The applications of complex networks have provided a new perspective for studying the mechanisms of these systems. In essence, the stock market is a typical complex network system since the vertices are stocks in the financial market and some vertices are connected by an edge if they have a relationship with each other. In the stock market, the price of stock fluctuates frequently with the dynamic evolution of the entire financial system. Previous studies suggest that a stock market network could be established based on the price correlations. From different angles, people provided many effective complex network construction methods such as minimum cost spanning tree $(M S T)$, planar maximally filtered graph $(P M F G)$, and correlation threshold method. Huang and Tse used the correlation threshold method to construct a stock correlation network in order to analyze the information of stock markets where the nodes are the stocks and the connections are determined by the threshold of Pearson correlation (PCC) $[1,2]$. Originally, Mantegna used the Pearson correlation and MST method to build a stock network and revealed the general hierarchical structure of the market [3]. Bonanno et al. also used the method and found that stock market networks present different hierarchical structures as the time horizon changes [4]. Tumminello et al. also used the correlation coefficient between stock price dynamics time series and the PMFG method to generate stock networks [5].

Since the stock price correlations are widely used in the popular methods mentioned above, the studies of dynamic correlations and relationships become crucial for constructing the stock market complex network and analyzing the economic features of the stock market. In previous studies, there are some conventional methods to quantify the correlations of stock price time series, such as Pearson correlation, cross-correlation, and canonical correlation. But it is known that financial data are highly nonstationary and the conventional methods may not suited for it [6]. 
Accordingly, it is important to investigate the time-migrated or time-asynchronous correlations. To investigate the time characters of financial series, Grey Relational Analysis [7], Detrended Fluctuation Analysis (DFA), and Detrended Cross-correlation Analysis (DCCA) [8] were proposed to quantify the long-range power-law correlations of nonstationary time series.

Previous research has established that DCCA provides a proper approach to quantitatively measure the long-range cross-correlation of nonstationary time series. It inspires us to investigate the time series of stock market using these methods. To the best of our knowledge, there is still little research to gain the insights into time-migrated or timeasynchronous correlations of the stock market price series. Therefore, we present a time-migrated DCCA correlation coefficient in this paper. We not only calculate the DCCA correlation coefficients of the stock market but also investigate the time-migrated relationships. In the field of stock market regulation, price limit policy is widely used to prevent stock prices from rising or falling too violently, especially during the stock crash period. Once stock prices hit the limit, they are not allowed to move beyond the limit. The price limit is supposed to give frenzied traders time to cool off and save the price from "falling off a cliff" [9]. Many of the stock exchanges adopt price limit to maintain the stability and curb the overreaction of the stock market [10]. But there are other voices that the price limit is ineffective and imposes serious costs to the stock market [11]. Rare literature of price limit refers to the econometric analysis of the stock market model. In that case, we also evaluate the effectiveness of the price limit from the perspective of complex network in this paper. In this context, this research aims to address the following three questions:

(1) How to mining the time-migrated correlations of the stock prices?

(2) What are the properties and community structures of the stock market from the perspective of complex network.

(3) How to evaluate the effectiveness of price limit reform on the stock market network.

To achieve this goal, we propose a time-migrated DCCA method and study the correlations of Chinese stock market. Lately, we apply the threshold method to construct stock market networks for analyzing the effectiveness of the price limit reform.

The paper is organized as follows. Section 2 presents dataset and methods employed in this study. Section 3 shows the properties and community structures of the stock market and provides effectiveness results of the price limit reform on stability and systemic risk. Section 4 concludes the paper. Finally, Data availability and References are also presented.

\section{Materials and Methods}

2.1. Stock Market Dataset. In this paper, we choose the CSI 300. The CSI 300 dataset contains the daily close prices of the 300 large-scale stocks with good liquidity. The CSI 300 index usually covers about $60 \%$ of the Shanghai Composite and Shenzhen Component. The dataset of CSI 300 stock index is from Mar 28th, 2012 to Mar 29th, 2019 (data source: Choice Financial Terminal) which contains 1703 daily close prices of the CSI 300 companies, including the 2015 market crash period. The samples of the close price series are presented in Table 1.

To get a more stable price series, we calculate the return price of the dataset by

$$
r_{i}(t)=\ln \left[\frac{p_{i}(t+1)}{p_{i}(t)}\right],
$$

where $r_{i}(t)$ is the return price of stock $i$ at the day $t, p_{i}(t+1)$ and $p_{i}(t)$ are the close prices of stock $i$ at the day $t$ and the day $t-1$. Thus, $i \in[1,300]$ and $t \in[1,1702]$. The daily return prices of the stock 000415 are presented in Figure 1. As is shown in Figure 1, the return price varies between [-1, 1] while the close price varies between [3.38, 28.28]. Thus, the return price has better properties to avoid the excessive influence and nonstationarity of the dataset.

In this paper, we obtain the DCCA cross-correlation coefficient and time-migrated DCCA cross-correlation coefficient with the return prices. For this purpose, we introduce a brief theoretical description of the two methods.

2.2. DCCA Cross-Correlation Coefficient. Traditionally, the DCCA cross-correlation coefficient is derived from DFA and DCCA $[6,12,13]$. The DFA method is a common method for investigating the long-range power-law self-correlations of single time series, and the DCCA method has demonstrated its usefulness to determine the long-range power-law cross-correlations of two nonstationary time series [14]. One step further, the DCCA cross-correlation coefficient is an effective method to quantify the level of cross-correlation between two nonstationary time series at different temporal scales [15-17]. The algorithm of DCCA correlation coefficient consists of five steps.

Step 1: Supposing that there are two stock price series $\xi(t)$ and $\eta(t)$, we obtained two removing mean and accumulated time sequences:

$$
\begin{aligned}
\xi^{\prime}(t) & =\sum_{i=1}^{t}[\xi(i)-\bar{\xi}], \\
\eta^{\prime}(t) & =\sum_{i=1}^{t}[\eta(i)-\bar{\eta}] .
\end{aligned}
$$

Where $\bar{\xi}$ and $\bar{\eta}$ are the mean value of time series $\xi(t)$ and $\eta(t), t=1,2, \ldots, T$.

Step 2: We cut both time sequences $\xi^{\prime}(t)$ and $\eta^{\prime}(t)$ into $(N-s)$ overlapping segments $\xi_{k}^{\prime}(t)$ and $\eta_{k}^{\prime}(t)$, with $k=$ $1, \ldots, T-s$ and $0 \leq s<T-1$. The length of $\xi_{k}^{\prime}(t)$ and $\eta_{k}^{\prime}(t)$ is $s+1$.

Step 3: we calculate the local trend of each_segment $\xi_{k}^{\prime}(t)$ and $\eta_{k}^{\prime}(t)$ by a least-squares fit method, $\xi_{k}(t)$ and $\eta_{k}(t)$. Then we define the detrended time series for each 
segment and calculate self-variance and covariance of each residual by:

$$
\begin{aligned}
& F_{\xi \xi}^{2}(i)=\frac{1}{s+1} \sum_{t=1}^{s+1}\left[\breve{\xi}_{i}(t)-\xi_{i}^{\prime}(t)\right]^{2}, \\
& F_{\eta \eta}^{2}(i)=\frac{1}{s+1} \sum_{t=1}^{s+1}\left[\breve{\eta}_{i}(t)-\eta_{i}^{\prime}(t)\right]^{2}, \\
& F_{\xi \eta}^{2}(i)=\frac{1}{s+1} \sum_{t=1}^{s+1}\left[\breve{\xi}_{i}(t)-\xi_{i}^{\prime}(t)\right]\left[\breve{\eta}_{i}(t)-\eta_{i}^{\prime}(t)\right] .
\end{aligned}
$$

Where $\breve{\xi}_{k}(t)$ and $\breve{\eta}_{k}(t)$ are the fitting polynomials of segment $k$. And $\xi_{i}(t)-\xi_{i}^{\prime}(t)$ and $\breve{\eta}_{i}(t)-\eta_{i}^{\prime}(t)$ are the detrended time series of the segment $i$, respectively.

Step 4: We obtain the detrended covariance function of all segments by

$$
\begin{aligned}
& F_{\mathrm{DFA}_{-} \xi}(s)=\left(\frac{1}{N-s} \sum_{i=1}^{N-s} F_{\xi \xi}^{2}(i)\right)^{(1 / 2)} \\
& F_{\mathrm{DFA}_{-} \eta}(s)=\left(\frac{1}{N-s} \sum_{i=1}^{N-s} F_{\eta \eta}^{2}(i)\right)^{(1 / 2)} \\
& F_{\mathrm{DCCA}}^{2}(s)=\frac{1}{N-s} \sum_{i=1}^{N-S} F_{\xi \eta}^{2}(i) .
\end{aligned}
$$

Step 5: Finally, we calculate the DCCA cross-correlation coefficient by

$$
\rho_{\mathrm{DCCA}}(s)=\frac{F_{\mathrm{DCCA}}^{2}(s)}{F_{\mathrm{DFA}_{-} \xi}(s) F_{\mathrm{DFA}_{-} \eta}(s)} .
$$

The DCCA cross-correlation coefficient is a function of the time segment length $s$. As we can see, the DCCA cross-correlation coefficient equals to Pearson cross-correlation coefficient when $s=0$. According to the Cauchy-Schwarz inequality, the DCCA crosscorrelation coefficient ranges $[-1,1]$. Like the Pearson cross-correlation coefficient, the value of $\rho_{\text {DCCA }}(s)=0$ means there is no correlation between the two time series. The $\rho_{\text {DCCA }}(s)=1$ means a full positive correlation, whereas $\rho_{\text {DCCA }}(s)=-1$ means a full negative correlation. A major advantage of $\rho_{\text {DCCA }}(s)$ is to measure the cross-correlations between two nonstationary time series at the different segment length $s$ [15]. It is more robust to contaminated noises and amplitude ratio than Pearson correlation [18]. There are a number of $\rho_{\text {DCCA }}$ applications in meteorology $[19,20]$, physiology $[21,22]$, economy $[13,23]$, financial $[14,16,24-26]$, and other research areas.
2.3. Time-Migrated DCCA Cross-Correlation Coefficient. Traditionally, the DCCA cross-correlation coefficient is implemented by measuring the correlations of each detrended segment synchronously. As is shown in equation (4), we use the equal-time segment $i$ of $\xi(t)$ and $\eta(t)$ in Figure 2(a) when we calculate $F_{\xi \eta}^{2}(i)$. However, it should be further noticed that the price series have not only synchronous relationships but also asynchronous relationships in real-time stock markets. For example, there is a lead-lag effect on the stock market, which means that stock prices of some firms show a delayed or ahead temporal evolution pattern to other firms' stock prices [27-30]. Since a possible delay between the stocks could be accounted in the time series, we consider the following case in Figure 2(b): supposing two time series $\xi(t)$ and $\eta(t)$ in the stock market, we calculate the detrended correlations of each segment $i$ in $\xi(t)$ and $\eta(t)$, but in some cases, the segment $i$ of $\xi(t)$ may have a relationship with segment $j$ of $\eta(t)$ in some cases. So we consider these asynchronous relationships in the time-migrated DCCA cross-correlation coefficient.

The algorithm of time-migrated DCCA correlation coefficient consists of the following seven steps:

Step 1: We calculate removing mean and accumulated time sequences of $\xi(t)$ and $\eta(t)$ :

$$
\begin{aligned}
\xi^{\prime}(t) & =\sum_{i=1}^{t}[\xi(i)-\overline{\bar{\xi}}], \\
\eta^{\prime}(t) & =\sum_{i=1}^{t}[\eta(i)-\bar{\eta}] .
\end{aligned}
$$

Where $\bar{\xi}$ and $\bar{\eta}$ are the mean value of time series $\xi(t)$ and $\eta(t), t=1,2, \ldots, T$.

Step 2: We cut both two time sequences $\xi^{\prime}(t)$ and $\eta^{\prime}(t)$ into $(N-s)$ overlapping segments $\xi_{k}^{\prime}(t)$ and $\eta_{k}^{\prime}(t)$, with $k=1, \ldots, T-s$ and $0 \leq s<T-1$. The length of $\xi_{k}^{\prime}(t)$ and $\eta_{k}^{\prime}(t)$ is $s+1$.

Step 3: For the detrended time series of the segment $i$, we calculate the time-migrated covariance of $\xi_{i}(t)-$ $\xi_{i}^{\prime}(t)$ and $\breve{\eta}_{j}(t)-\eta_{j}^{\prime}(t)$, where $i, j=1, \ldots, T-s$.

$$
F_{\xi \eta}^{2}(i, j)=\frac{1}{s+1} \sum_{t=1}^{s+1}\left[\breve{\xi}_{i}(t)-\xi_{i}^{\prime}(t)\right]\left[\breve{\eta}_{j}(t)-\eta_{j}^{\prime}(t)\right] .
$$

Step 4: We find the max value of $F_{\xi_{\eta}}^{2}(i, j)$ for $\xi^{\prime}(t)$ by

$$
F_{\max _{-} \xi \eta}^{2}(i)=\max _{1 \leq j \leq N-s} F_{\xi \eta}^{2}(i, j) \text {. }
$$

Where $i, j=1, \ldots, T-s$ and $j^{*}$ is the argument that makes the equation (9) true:

$$
j^{*}(i)=\underset{1 \leq j \leq N-s}{\operatorname{argmax}} F_{\xi \eta}^{2}(i, j) .
$$


Step 5: We calculate the detrended self-variance in each segment $i$ of $\xi(t)$ by

$$
F_{\xi \xi}^{2}(i)=\frac{1}{s} \sum_{t=1}^{s+1}\left[\zeta_{i}(t)-\xi_{i}^{\prime}(t)\right]^{2} .
$$

According to the Cauchy-Schwarz inequality, we calculate detrended self-variance in the segment $j^{*}(i)$ of $\eta(t)$ :

$$
F_{\eta^{*} \eta^{*}}^{2}(i)=\frac{1}{s} \sum_{t=1}^{s+1}\left[\breve{\eta}_{j^{*}(i)}(t)-\eta_{j^{*}(i)}^{\prime}(t)\right]^{2} .
$$

Step 6: We obtain the time-migrated detrended covariance function $F_{t m-\mathrm{DCCA}}^{2}(s), F_{\mathrm{DFA}_{-} \xi}(s)$ and $F_{\mathrm{DFA}_{\eta}}(s)$ by

$$
\begin{aligned}
F_{t m-\mathrm{DCCA}}^{2}(s) & =\frac{1}{N-s} \sum_{i=1}^{N-s} F_{\text {max }_{-} \xi \eta}^{2} \\
F_{\mathrm{DFA}_{-} \xi}(s) & =\left(\frac{1}{N-s} \sum_{i=1}^{N-S} F_{\xi \xi}^{2}(i)\right)^{(1 / 2)}, \\
F_{\mathrm{DFA}_{-} \eta^{*}}(s) & =\left(\frac{1}{N-s} \sum_{i=1}^{N-S} F_{\eta^{*} \eta^{*}}^{2}(i)\right)^{(1 / 2)} .
\end{aligned}
$$

Step 7: Finally, we calculate the time-migrated DCCA cross-correlation coefficient by

$$
\rho_{t m-\mathrm{DCCA}}(s)=\frac{F_{t m-\mathrm{DCCA}}^{2}(s)}{F_{\mathrm{DFA}_{-} \xi}(s) F_{\mathrm{DFA}_{-} \eta^{*}}(s)} .
$$

We calculate the relationships asynchronously in the time-migrated DCCA cross-correlation coefficient. We get the maximum of $F_{\xi \eta}^{2}(i, j)$ to detect the time-migrated relationships of two stocks in order to maximize the correlation detection ability. According to the Cauchy-Schwarz inequality, the time-migrated DCCA cross-correlation coefficient is also a set of dimensionless coefficient ranging from -1 to 1 . A higher value of DCCA cross-correlation coefficient means a closer relationship with each other.

The DCCA cross-correlation coefficient provides a proper approach to measure equal-time relationships between two nonstationary time series, but the time-migrated DCCA cross-correlation coefficient is also available for measuring relationships between two nonstationary time series with asynchronous relationships. They are both dimensionless coefficients that can be compared with other nondimensional methods, such as Pearson coefficients.

2.4. Stock Market Network Model. In the upper subsection, we studied the equal-time DCCA cross-correlation coefficient and the time-migrated DCCA cross-correlation coefficient. It is now well established from a variety of studies that a network could be constructed from the Pearson correlation coefficient matrix of the complex system
$[1,2,4,31-33]$. In this section, we apply the threshold method to construct stock market networks with these two methods. Additionally, we compare the topology properties and community structures of the stock networks. First we calculate the equal-time DCCA cross-correlation coefficient $\rho_{\text {DCCA }}^{i j}(s)$ and time-migrated DCCA cross-correlation coefficient $\rho_{t m-D C C A}^{i j}(s)$ of the entire return price pairs in the dataset on a different time scale $s$. Then we obtain the maximum value when $s=s^{*}$ by

$$
\begin{aligned}
\rho_{\text {DCCA_max }}^{i j} & =\max _{s=s^{*}} \rho_{\text {DCCA }}^{i j}(s), \quad i, j \in[1,300], \\
\rho_{t m \text {-DCCA_max }}^{i j} & =\max _{s=s^{*}} \rho_{t m \text {-DCCA }}^{i j}(s), \quad i, j \in[1,300],
\end{aligned}
$$

where $\rho_{\text {DCCA_max }}^{i j}$ and $\rho_{t m-D C C A \_m a x}^{i j}$ are the max coefficients between stocks $i$ and $j$.

Then a metric distance of stock $i$ and $j$ can be translated into connection weight by $[3,4,32]$

$$
\begin{aligned}
D_{\mathrm{DCCA}}^{i j} & =\sqrt{2\left(1-\rho_{\mathrm{DCCA} \_ \text {max }}^{i j}\right)}, \\
w_{\mathrm{DCCA}}^{i j} & =\frac{1}{D_{\mathrm{DCCA}}^{i j}}, \\
D_{t m-\mathrm{DCCA}}^{i j} & =\sqrt{2\left(1-\rho_{t m-\mathrm{DCCA} \_\max }^{i j}\right)}, \\
w_{t m-\mathrm{DCCA}}^{i j} & =\frac{1}{D_{t m-\mathrm{DCCA}}^{i j}} .
\end{aligned}
$$

In both cases, we get $300 \times 300$ matrix of connection weights $W_{\text {DCCA }}$ and $W_{t m-\text { DCCA }}$.

Finally, we set a certain threshold value $\theta$ to construct the stock market network. Let the Graph $G=(V, E)$ represents stock market network, where the node $v_{i} \in V$ represents stock $i$ and edge $e_{i j} \in E$ represents the connections of the stock $i$ and stock $j$. The set of connections is established by

$$
E= \begin{cases}e_{i j}=1, & i \neq j \text { and } w_{i j}>\theta, \\ e_{i j}=0, & i=j .\end{cases}
$$

The complex network construction algorithm is given by Algorithm 1. We get different connection topologies with different values of threshold $\theta$.

\section{Results and Discussion}

In this study, we construct the stock market complex network based on the threshold method with two sets of coefficient matrices. Previous research has established certain applications of the complex network in economics: relationships [34], contagion [35-37], risk [38-41] and so on $[42,43]$, but few studies are based on the policy effect. So in order to quantify the effectiveness of price limit reform, we first analyze statistical characteristics of the coefficients and network properties; then we make an econometric analysis about the bailout strategy such as price limit reform base on the stock market network in this section. 
TABLE 1: Close prices of three stocks in four trading days.

\begin{tabular}{lcccccc}
\hline Stock code & Major business & May 13, 2016 & May 16, 2016 & May 17, 2016 & May 18, 2016 & May 19, 2016 \\
\hline 000100 & Electronic equipment & 3.36 & 3.38 & 3.36 & 3.3 & 3.3 \\
000157 & Special equipment & 4.12 & 4.13 & 4.11 & 4.06 & 4.05 \\
000166 & Securities service & 8.06 & 8.08 & 8.06 & 8.07 & 8.03 \\
\hline
\end{tabular}

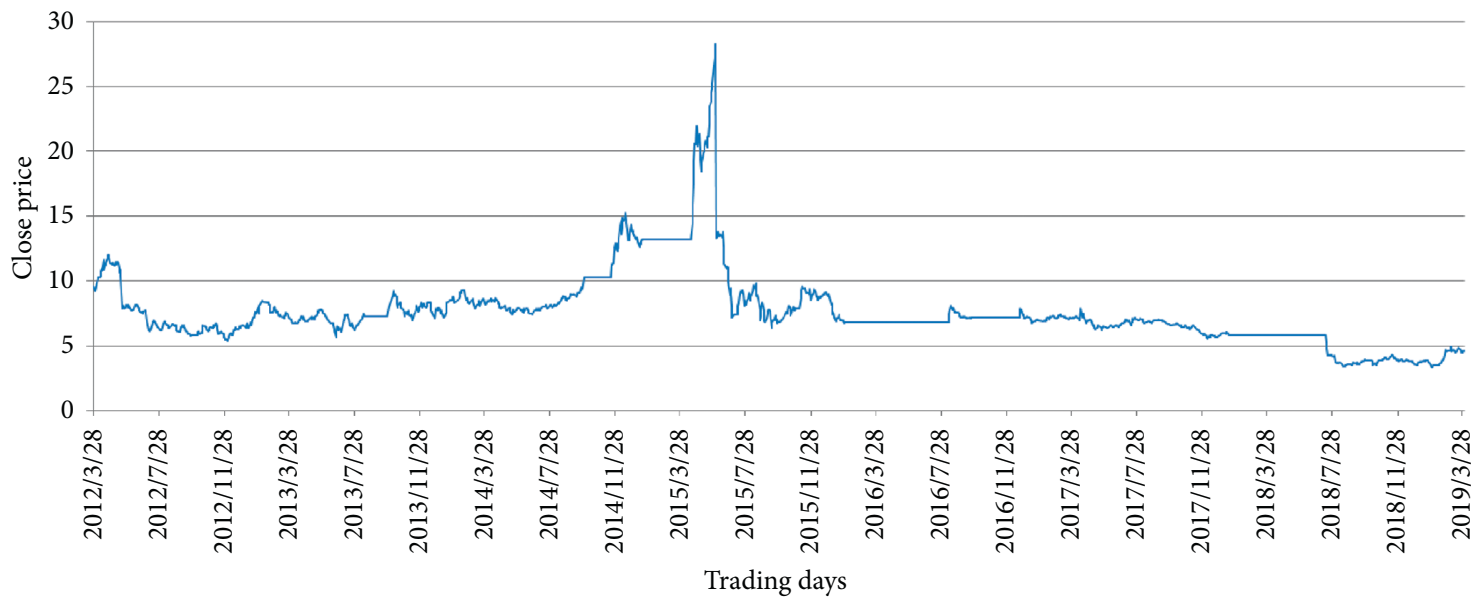

(a)

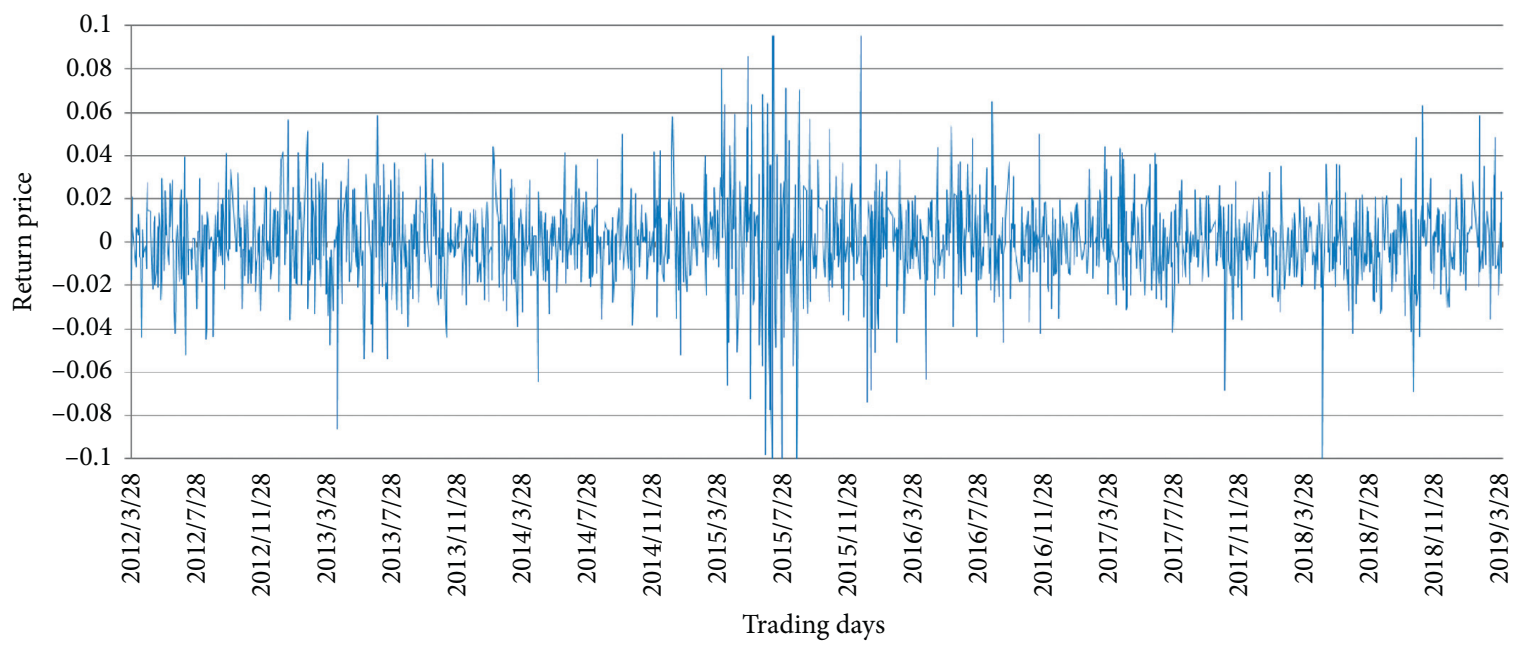

(b)

FIgURE 1: Closing prices and return prices of stock 000415.

3.1. Statistical Analysis. Based on the methods and dataset of Section 2, we calculate the correlation matrices with the two methods and examine the distributions of the empirical,

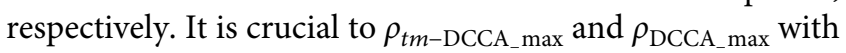
proper values of time segment length $s$. As is shown in Figure 3 , a clear spike of $\rho_{t m \text {-DCCA max }}$ and $\rho_{\text {DCCA max }}$ appears with smaller value of $s$ and the curves tend to flat as $s$ increases. So we choose segment length $1 \leq s \leq 15$ to analyze the relatively short term correlation coefficients.

We display the statistical results of DCCA and timemigrated DCCA in contrast to the PCC in Figure 4 and Table 2. The details of PCC coefficient could be obtained in
$[1,31]$. It can be seen in Figure 4 that the $\rho_{\text {DCCA }}$ distribution plot has a shape similar to the $\rho_{\text {PCC }}$ distribution, and the time-migrated DCCA has a bigger mean value and kurtosis value than DCCA and PCC. It tells us we get a bigger correlation in most cases and the distribution is more concentrated. The time-migrated DCCA method could ensure more relevant results than the other two methods. In Figure 4, it is easy to see a more concentrated distribution of time-migrated DCCA which is more sensitive to the changing of threshold $\theta$. As a result, the constructed network based on the time-migrated DCCA is more representative with threshold $\theta$. 


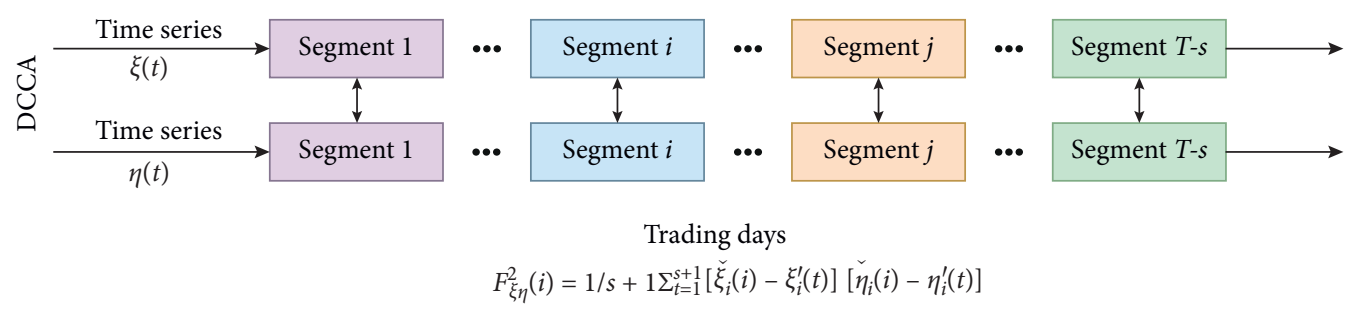

(a)

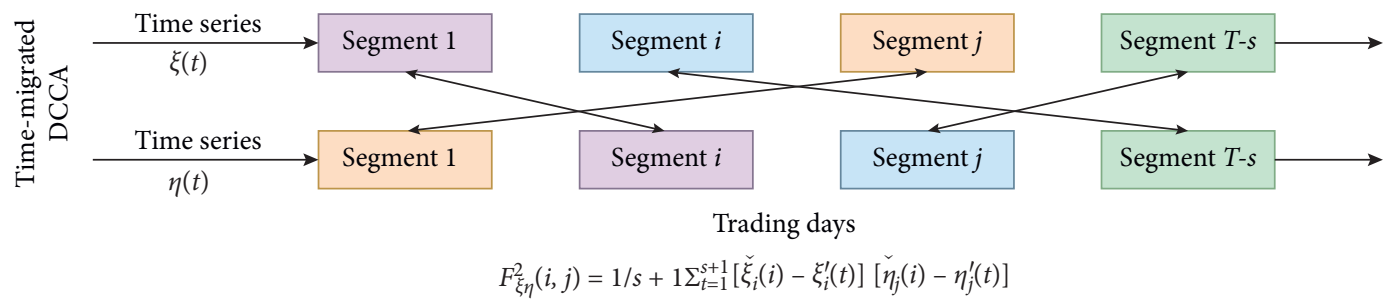

(b)

Figure 2: (a) Principles of DCCA cross-correlation coefficient (synchronous correlations). (b) The possible scenario of time-migrated DCCA cross-correlation coefficient (asynchronous correlations).

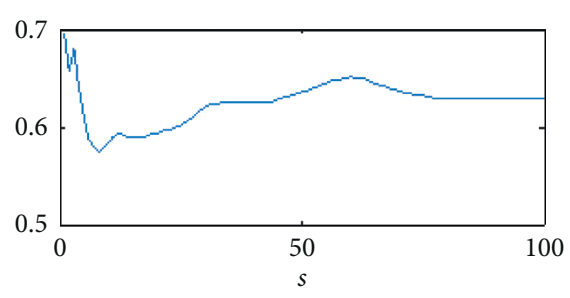

(a)

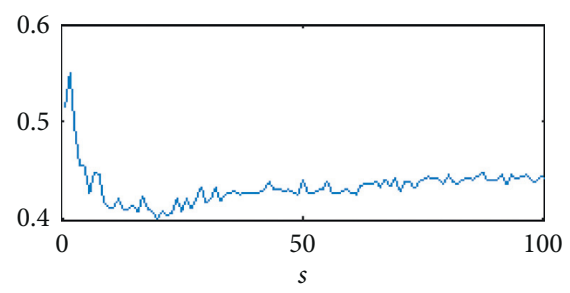

(d)

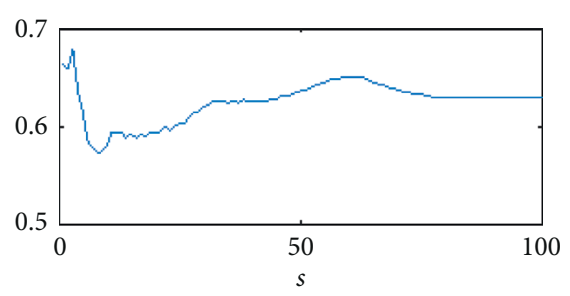

(b)

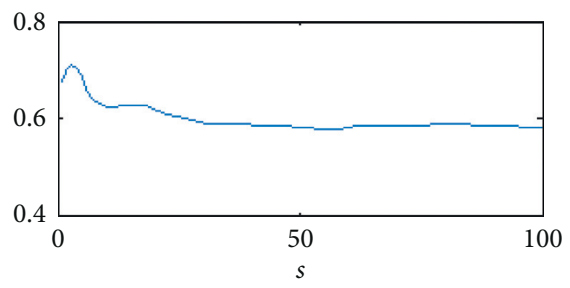

(e)

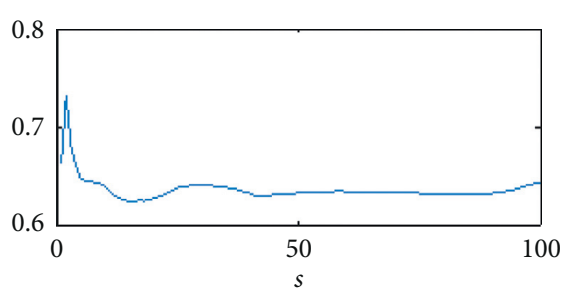

(c)

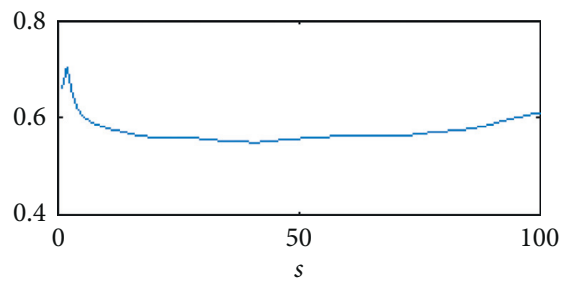

(f)

FIGURE 3: Some samples of $\rho_{\text {tm-DCCA_max }}$ and $\rho_{\text {DCCA_max }}$ at different time scales $s$.

Table 2 compares an overview of the three coefficients. It can be seen from Table 2 that statistics of DCCA and PCC are more similar to each other. The mean value of timemigrated DCCA is larger than DCCA and PCC, which means time-migrated DCCA detects more relationships of the dataset. The maximum of time-migrated DCCA is smaller than DCCA and PCC and the minimum is larger, which means the time-migrated DCCA has a smaller range. The smaller STD means a higher level data concentration of time-migrated DCCA and more sensitive to the threshold $\theta$.

\subsection{Network Properties and Community Structure}

3.2.1. Evaluation of Coefficients. The next section of the survey is concerned with network properties. First we analyze the giant component of the network with a different threshold $\theta$. The giant component is an important quantity representing the largest fraction of the complex network, which is a measurement of the network effectiveness [44]. In Figure 5, we can see that the giant components of the DCCA stock network and time-migrated network decrease as the threshold $\theta$ increases. Especially when threshold $\theta$ increases from 1.32 to 2 , the giant component of time-migrated DCCA network drops from 0.98 to 0.02 sharply. It is because most of the $\rho_{\mathrm{TM}-\mathrm{DCCA}}$ are distributed in this range. As a result, the stock market networks are scale-free. In addition, we introduce the dataset of Shanghai and Shenzhen A-shares (2016-2018) to testify the applicability of the stock network model. We find that the stock market network is still scalefree and these statistics are also available for further research. We think that the network model remains robust across different periods and datasets. 


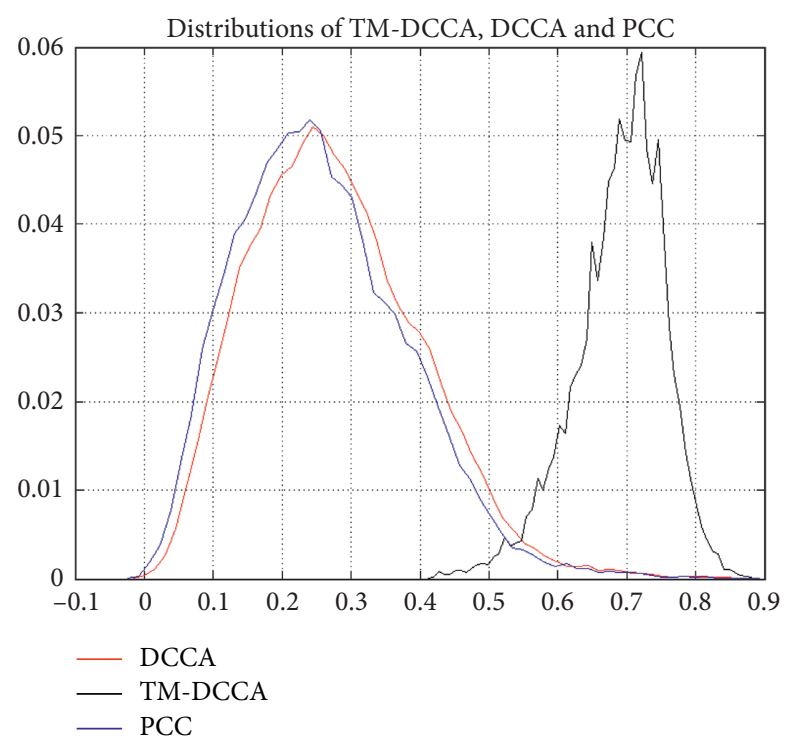

FIgURE 4: Distributions of time-migrated DCCA, DCCA and PCC coefficients.

Input: An empty complex network graph of stock market $G=(V, E)$, The sets of the stock market nodes $v_{i} \in V$, The weight matrix of the stock market $w_{i j} \in W$, The connection matrix of the stock market $e_{i j} \in E$, The threshold $\theta$, where $i, j=1, \ldots, N$; Output: The complex network graph of stock market $G(V, E)$;

(1) for each $v_{i} \in V$ do

(2) add node $v_{i}$ to $V$ and update $G=(V, E)$

(3) end

(4) for each $e_{i j}$ and $w_{i j}$ do

(5) if $i \neq j$ and $\mathbf{w}_{\mathrm{ij}}>\theta$ then

(6) set $e_{i j}=1$ and add edge $e_{i j}$ to $E$

(7) update $G=(V, E)$;

(8) end

(9) end

Algorithm 1: Complex network construction algorithm on stock market.

To construct the stock market network, we need a proper threshold $\theta$ to determine the edge connectivity of the nodes. We divide all the $\rho_{\text {TM-DCCA }}$ and $\rho_{\text {DCCA }}$ into three conditions according to different thresholds by Table 3 , suggested in [15]. In this paper, we consider that the pair of nodes have strong correlations if the $\rho_{\text {TM-DCCA }}$ has a greater value (more than 1.62). Here we choose the threshold $\theta_{\mathrm{TM}-\mathrm{DCCA}}=1.62$ and $\theta_{\mathrm{DCCA}}=1.25$ to construct the stock networks, which represents the stock network with connections. Therefore, about $33 \%$ of total nodes are included in the stock networks according to the threshold $\theta_{\mathrm{TM}-\mathrm{DCCA}}=1.62$ and $\theta_{\mathrm{DCCA}}=1.25$. Other isolated nodes are removed. Then, the connected nodes of the stock works are 106 and 101, the number of connections are 646 and 203. Finally, the average node degree is 12.189 and 4.02 , average clustering coefficient is 0.066 and 0.77 . With the threshold $\theta$ defined, we describe the network parameters in Table 4. The average degree of time-migrated DCCA network is much bigger than DCCA. In the time-migrated DCCA network, a smaller community has a bigger average degree which means that stocks in a smaller community have denser connections with each other.

3.2.2. Evaluation of Coefficients. In this section, we wish to compare the community structure of the stock market network. It helps us to analyze the relationships and network structure for further research. We apply the algorithm of Blondel to detect communities of the stock network which has been widely used in complex network analysis [45]. There are several advantages of the Blondel algorithm. The algorithm is a heuristic method that is fast and good for 


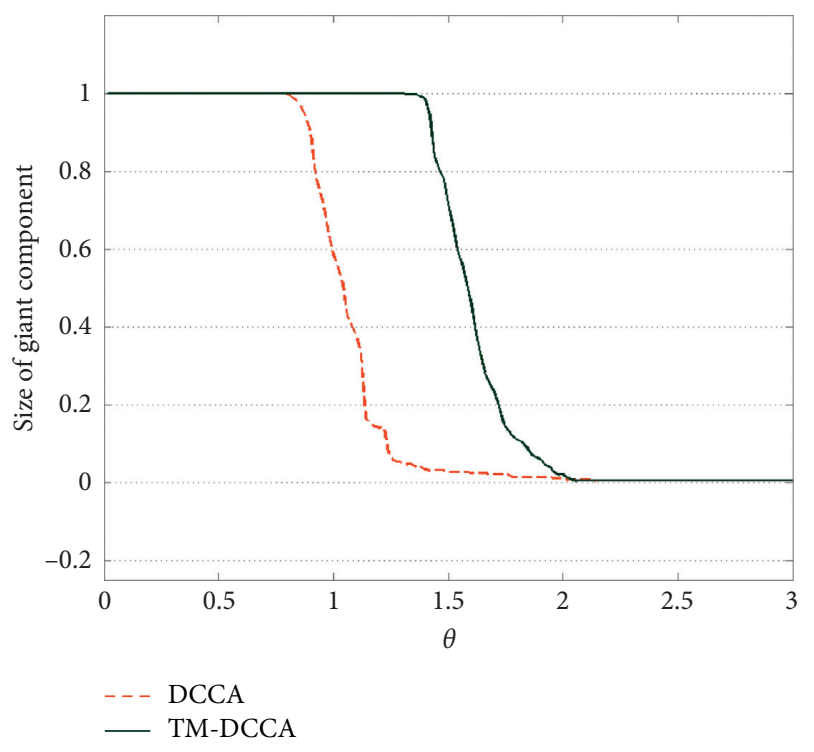

FIGURE 5: Giant components of time-migrated DCCA and DCCA coefficients.

TABLE 2: Statistical analysis of Time-migrated DCCA, DCCA, and PCC.

\begin{tabular}{lccc}
\hline Statistics & Time-migrated DCCA & DCCA & PCC \\
\hline Mean & 0.6892 & 0.2769 & 0.2572 \\
Max & 0.8803 & 0.8900 & 0.8918 \\
Min & 0.4116 & -0.0159 & -0.0241 \\
STD & 0.0659 & 0.1234 & 0.1227 \\
Skewness & -0.6612 & 0.5650 & 0.6173 \\
Kurtosis & 3.7184 & 3.4296 & 3.5463 \\
\hline
\end{tabular}

TABLE 3: Correlation conditions of time-migrated DCCA and DCCA.

\begin{tabular}{lccc}
\hline Correlation condition & Weak & Medium & Strong \\
\hline Giant component & $1.0-0.7$ & $0.7-0.4$ & $0.4-0.02$ \\
$\rho_{\text {TM-DCCA }}$ & $1.32-1.5$ & $1.5-1.62$ & $1.62-2.0$ \\
$\rho_{\text {DCCA }}$ & $0.78-0.96$ & $0.96-1.08$ & $1.08-1.76$ \\
\hline
\end{tabular}

large-scale networks. It is shown to outperform all other known community detection methods in terms of computation time in literature [45]. The algorithm is divided into two phases that are repeated iteratively. The first phase repeats the nodes allocation process until the maximum of the modularity is attained. The second phase consists in building a new network whose nodes are in the communities found during the first phase. A separating layout is used to reveal communities in stock market networks. As is shown in Figures 6-7, different node colors represent different communities and node label sizes reflect the node degree. Intuitively, nodes in the same community are stocks belonging to the same industry classification in the DCCA stock network in Figure 6. That matches our expectations. It is because the stock companies belonging to the same industry classification interact more frequently with each other and usually have closer relationships in reality. The communities of time-migrated DCCA are density connected in comparison with DCCA.

The statistic details are shown in Table 5. We present 5 main communities in the DCCA network while 3 main communities in time-migrated DCCA in Table 5. We can see that the community sizes of time-migrated DCCA network are larger than DCCA. What stands out in the table is that the major business of DCCA network concentrated in one or two fields, but the major business of time-migrated DCCA network distributed in more than six fields. These findings suggest that the time-migrated DCCA network contains more relevant information than the DCCA network. Intuitively, the stock market networks can reflect important properties of the real stock market. This inspires us of a new idea: the stock network model could be helpful to analyze the effectiveness of the stock market policy according to the dynamic evolution process of the complex network system. Denser connections could give us more information about the relationships. Thus, we use the time-migrated DCCA stock network model to conduct our analysis on the effectiveness of price limit policy (Table 6).

3.3. Simulation Results of Price Limit. In this section, we attempt to provide some insight into the effectiveness of price limit by simulating the dynamic evolution of timemigrated DCCA stock network model. After evaluating the market stability and risk level under price limit and other situations, we find that the price limit has different effects at different stages of stock network evolution.

\subsubsection{Experimental Indicators}

May-Wigner Stability Theorem. May established a model for measuring the stability of a large complex ecosystem [46]. The theorem was approved and improved by researchers $[47,48]$. The May-Wigner Stability theorem is used to investigate the stability of the financial system such as stock market complex systems $[49,50]$. As a generalized stability indicator, the May-Wigner stability theorem is defined by 3 permanents: the size of the network $N$, the density of connections $D$, and the average interaction strength $a$ [50].

$$
N S=\sqrt{N D} a,
$$

where NS represents the network stability. The system is considered stable when $N S<1$ and a smaller value of $N S$ means the network is more stable. By definition, the density of connections $D$ (graph density) and average interaction strength $a$ (average node degree) in our research are given by

$$
\begin{gathered}
D=\frac{m}{c_{N}^{2}}, \\
a=\frac{m}{N},
\end{gathered}
$$

where $m$ is the number of connections and $C_{N}^{2}$ is the maximum number of possible connections. The network stability factor has the following formula: 


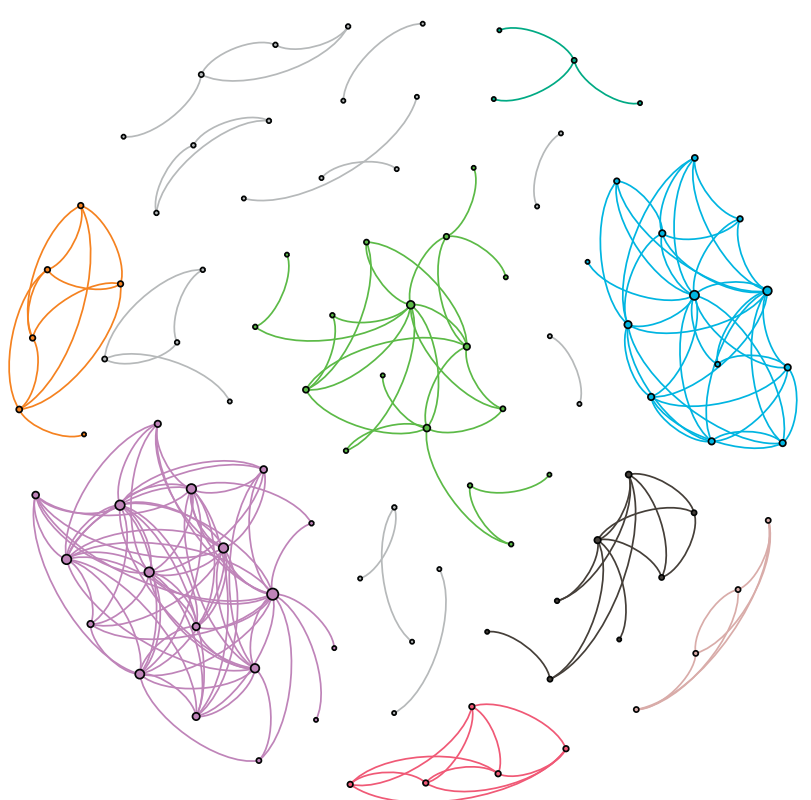

Figure 6: Topology graph of the DCCA stock network. Different node colors represent different communities and node and label sizes reflect the node degree.

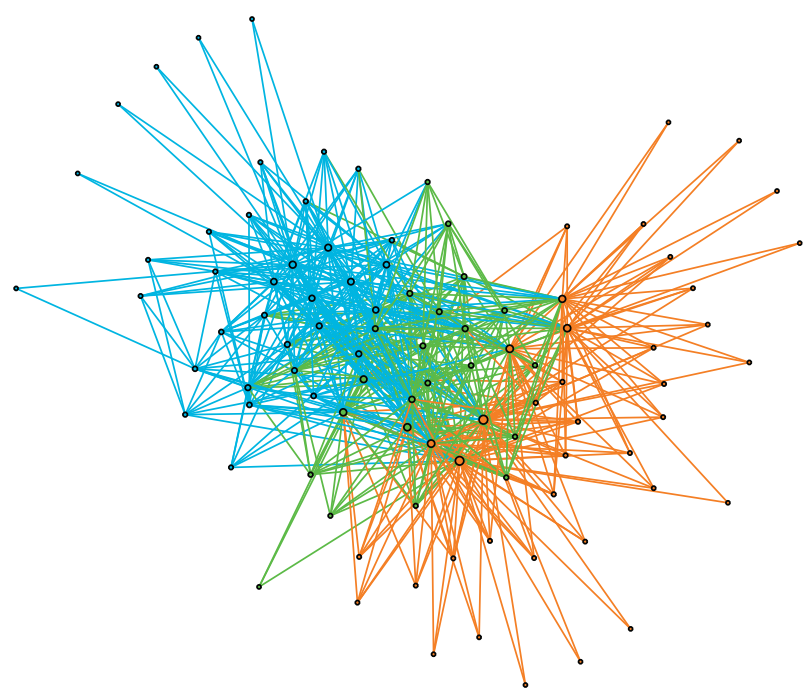

Figure 7: Topology graph of the time-migrated DCCA stock network. Different node colors represent different communities and node label sizes reflect the node degree.

TABLE 4: Network parameters of time-migrated DCCA and DCCA stock networks.

\begin{tabular}{lcc}
\hline Parameters & $\theta_{\text {TM-DCCA }}=1.62$ & $\theta_{\text {DCCA }}=1.25$ \\
\hline Number of nodes & 300 & 300 \\
Connected nodes & 106 & 101 \\
Number of connections & 646 & 203 \\
Average node degree & 12.189 & 4.02 \\
Graph density & 0.116 & 0.04 \\
Graph diameter & 4 & 6 \\
Average path length & 2.283 & 1.796 \\
Average clustering coefficient & 0.066 & 0.77 \\
\hline
\end{tabular}

$$
N S=\sqrt{N D} a=\sqrt{N \frac{m}{c_{N}^{2}}} \frac{m}{N}=\frac{m^{(3 / 2)}}{N \sqrt{N-1}}
$$

So we can calculate the network stability $N S(t)$ at time $t$.

In this study, we focus on the dynamic changes of the stock network. If the $N S(t)$ changes rapidly, it means that the stability of the stock network varies dramatically, which represents a more volatile market. In order to measure the instability of the complex network, we define a dimensionless evaluation factor stability variation $S V$ as follows:

$$
S V=\frac{\sqrt{\left(\sum_{t=1}^{n}(N S(t)-\overline{N S(t)}) / n\right)}}{\overline{N S(t)}}
$$

where $N S(t)$ is the network stability of time $t$ and a smaller value of $S V$ means a milder stability fluctuation.

Systemic Risk Evaluation. An important area of risk management is the systemic risk evaluation. Studying the correlation coefficient matrix is an important topic of systemic risk evaluation [51]. We perform eigenvector technique on the stock network to measure the systemic risk. Using this approach, researchers have been able to evaluate the risk contributions of the stocks and calculate the systemic risk $[44,52,53,54,55]$. In this study, we evaluate risk contributions based on the eigenvector centrality:

$$
R_{i}=\frac{1}{\lambda} \sum_{j=1}^{N} R_{j} g_{i j}, \quad i, j=1, \ldots, N
$$

where $R_{i}$ is the risk contribution of stock $i$, which is defined to be proportional to the weighted sum of all the stocks connected to stock $i$. $N$ is the total number of nodes in the stock network. $g_{i j}$ is the element of correlation coefficient matrix $G$, which represents the $\rho_{\mathrm{TM}-\mathrm{DCCA}}$ of stock $i$ and stock $j$. It could be also expressed as in matrix form according to the eigenvector centrality theory:

$$
G \vec{R}=\lambda \vec{R}
$$

where $\lambda$ is the eigenvalue corresponding to the eigenvector. Then we compute the average of the risk contribution of all the stocks in the network and obtain the systemic risk of the whole stock market network at time $t$.

$$
N R(t)=\frac{1}{N} \sum_{i=1}^{N} R_{i}, \quad i=1, \ldots, N .
$$

And we evaluate the systemic risk under each conditions by

$$
N R=\frac{1}{T} \sum_{t=1}^{T} N R_{t}, \quad t=1, \ldots, T
$$

3.3.2. Simulation Results. The fluctuations of the stock prices play an important role in the price discovery process, which provides crucial information on economic [56]. From 
TABle 5: Community Properties of DCCA stock market network.

\begin{tabular}{lccccc}
\hline $\begin{array}{l}\text { DCCA communities } \\
\left(\theta_{\text {DCCA }}=1.25\right)\end{array}$ & Community A & Community B & $\begin{array}{c}\text { Community } \\
\text { C Community D }\end{array}$ & Community E \\
\hline Nodes & 18 & 17 & 13 & 8 & 6 \\
Major business & Securities service (17), & Mining industry (15), & Banking (13) & Civil & Aerospace \\
Edges & pharmaceutical industry (1) & special equipment (2) & 39 & 12 & equipment (6) \\
Average node degree & 77 & 26 & 6.000 & 3.000 & 11 \\
Average path length & 8.56 & 3.059 & 1.5 & 1.71 & 3.667 \\
Graph density & 1.49 & 2.58 & 0.500 & 0.429 & 0.733 \\
\hline
\end{tabular}

TABLE 6: Community properties of the time-migrated DCCA stock market network.

\begin{tabular}{|c|c|c|c|}
\hline $\begin{array}{l}\text { Time-migrated DCCA } \\
\text { communities } \\
\left(\theta_{\mathrm{TM}-\mathrm{DCCA}}=1.62\right)\end{array}$ & Community A & Community B & Community $\mathrm{C}$ \\
\hline Nodes & 48 & 36 & 22 \\
\hline Major business & $\begin{array}{l}\text { Securities service (9), banking (8), } \\
\text { manufacturing (7), mining industry } \\
\text { (4), pharmaceutical industry (3), real } \\
\text { estate (3), energy industry (3), } \\
\text { chemical industry (2), others (9) }\end{array}$ & $\begin{array}{l}\text { Manufacturing (9), pharmaceutical } \\
\text { industry (4), securities service ( } 3) \text {, } \\
\text { banking (2), real estate (2), } \\
\text { transportation (2), information } \\
\text { technology (2), civil engineering (2), } \\
\text { others (10) }\end{array}$ & $\begin{array}{l}\text { Mining industry (5), securities } \\
\text { service (3), transportation (3), } \\
\text { information technology (3), } \\
\text { banking (2), others (6) }\end{array}$ \\
\hline Edges & 132 & 163 & 85 \\
\hline Average node degree & 5.5 & 7.7 & 9.5 \\
\hline Average path length & 2.24 & 1.72 & 1.97 \\
\hline Graph density & 0.117 & 0.368 & 0.259 \\
\hline
\end{tabular}

the perspective of price limit, the stock price is frenzied and the price discovery ability is weakened if a stock hits price limit $[9,11]$. It leads to market failure somehow. At this point, we assume such an evaluation model: if the stock price $i$ hits the price limit as it loses functionality, then node $i$ will be removed from the network.

The simulation experiment is arranged as follows. First we calculate the stability factor and systemic risk factor $N S(t)$ at time $t(t=1, \ldots, T)$. And we enhance price limit to analyze effectiveness of the time limit. Then we set the target removing and random removing strategy as the control group. Details of targeted removing and random removing strategy could be obtained in $[57,58]$. Finally, we get the results of stability analysis and systemic risk analysis.

Figure 8 presents an overview of $N S(t)$ of the time scale $t$ for the four situations. Generally, we notice that all the stability factors exhibit a downward trend as evolution of the network because the number of nodes decreases faster than the edges. Especially we can see the following:

Targeted Removal: The nodes of the stock network are removed in accordance with the sequence from the biggest degree value to the smallest, which is called targeted removal of the most important nodes [58]. From Figure 8, we can see that the network stability of targeted removal curve (green line) drops off sharply at the begging of the time step. Thus, this result indicates that the stock network is extremely vulnerable to targeted removal of the most important nodes.

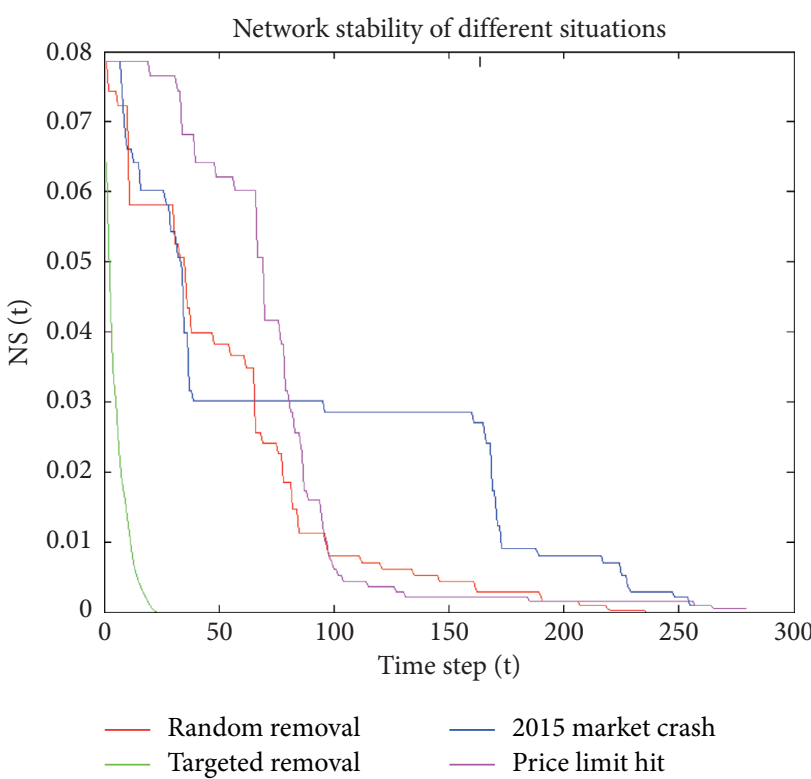

FIGURE 8: Network stability $N S(t)$ in different situations.

Random Removal: The nodes of the stock network are removed in a random order. Figure 8 shows that network stability factor of random removal (red line) has a relatively milder dynamic pattern compared to targeted removal. This indicates that the stock network is resistant to random removal. 


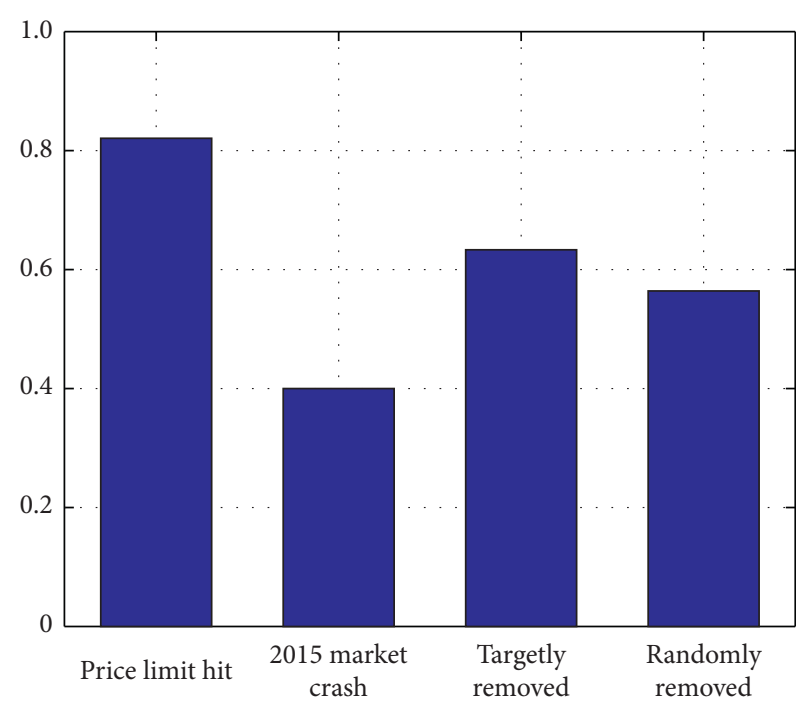

Figure 9: $S V$ of different situations.

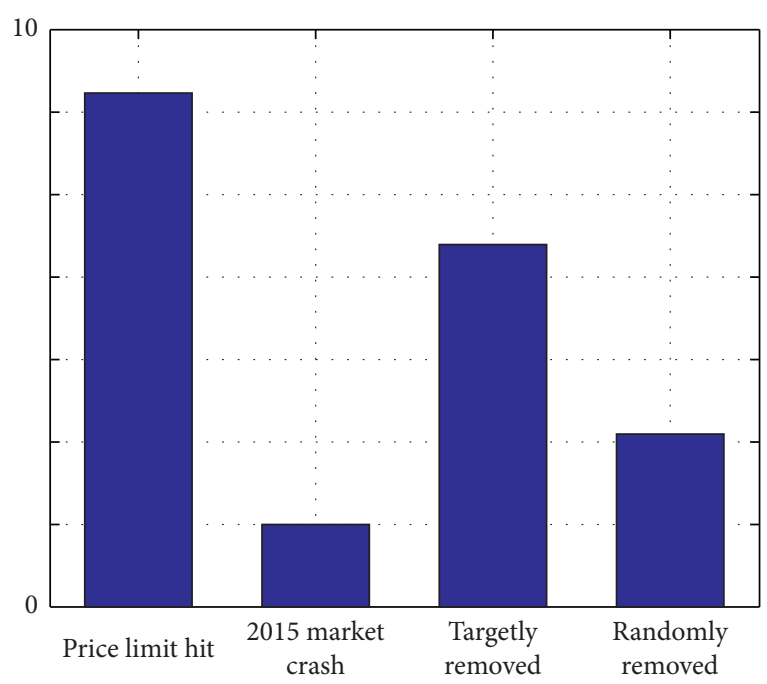

FIgURE 10: Systemic risk at different situations.

2015 Market Crash: The 2015 stock market began in June and lasted until August. About one thousand stocks hit the price limit. The stock market lost approximately 30 percent around the market crash. For the market crash simulation, we remove the node $i$ if the stock $i$ hits the price limit (10\%) during the 2015 market crash. We can see that (blue line) the network factor of market crash drops at the beginning and a platform zone appears in the middle time, reflecting the actual situation of actual situation.

Price Limit Hit: In contrast to stock with $10 \%$ price limit, the price limit is set to $5 \%$ for investigating the network stability performance with an enhanced price limit level. In Figure 8, we find that the price limit hit (magenta line) drops more gently than market crash in the beginning, which means the network stability. Then the network stability factor of price limit hit presents a more precipitous drop than market crash afterward. It means that the price limit hit could prevent violent variation, but also may lead to more violent variations afterwards.

In this case, it is possible to conclude that the cooling-off effect is significant when the price limit is first implemented, but the magnet effect also exists at the same time which takes the dominant in later time steps.

Figure 9 provides the normalized $S V$ of different situations. Actually, price limit group indicates more the stability changes on average than the other three groups. It means that the price limit may cause stability changes in the stock market.

Figure 10 presents the systemic risk $N R$ to compare the difference of the four situations. From Figure 10, we can see that systemic risk of targeted removal is higher than the random removal. And the price limit has the largest systemic risk. From the statistical results of systemic risk evaluation, we draw the conclusion that changing price limit has an effect on the market stabilization, and the systemic risk increases if the price limit is enhanced.

\section{Conclusion}

In this paper, we investigated the effectiveness of price limit on stock market based on the correlation study and complex network technology. Firstly, we proposed a time-migrated DCCA cross-correlation coefficient based on the DCCA cross-correlation coefficient. The time-migrated DCCA cross-correlation coefficient is suitable for nonstationary time series and detecting the time-migrated correlations, which ensure more relevant results than the DCCA method. Furthermore, we apply the threshold method to construct the stock networks and compare the topology properties and community structure of the stock network. We find that the time-migrated DCCA and DCCA stock network has different statistical properties and communities structures. And this fact has given us the opportunity to study the effectiveness of the price limit, especially during the stock market crash period. Finally, we simulated the dynamic evolution of the stock network under different situations. An interesting finding is that the price limit has different effects at different stages of evolution. We draw the conclusion that changing the price limit has an effect on market stabilization and the systemic risk, and the market stabilization will be lowered and the systemic risk will be increased if we enhance the price limit. We believe that such studies are relevant for a better understanding of the stock market and may lead to a better insight into the policy influence on the stock markets in further work. For example, the stock market network model could be helpful to evaluate the price limit performance in different situations. It may also contribute to risk management and stability regulation, which has a significant contribution to the stock market in reality.

\section{Data Availability}

All data used in this study are available from the Choice Financial Terminal and http://choice.eastmoney.com. 


\section{Conflicts of Interest}

The authors declare that there are no conflicts of interest.

\section{Acknowledgments}

This work was supported by National Natural Science Foundation of China (61673247), and the Research Fund for Distinguished Young Scholars and Excellent Young Scholars of Shandong Province (JQ201719).

\section{References}

[1] W.-Q. Huang, X.-T. Zhuang, and S. Yao, "A network analysis of the Chinese stock market," Physica A: Statistical Mechanics and Its Applications, vol. 388, no. 14, pp. 2956-2964, 2009.

[2] C. K. Tse, J. Liu, and F. C. M. Lau, "A network perspective of the stock market," Journal of Empirical Finance, vol. 17, no. 4, pp. 659-667, 2010.

[3] R. N. Mantegna, "Hierarchical structure in financial markets," The European Physical Journal B, vol. 11, no. 1, pp. 193-197, 1999.

[4] G. Bonanno, F. Lillo, and R. N. Mantegna, "High-frequency cross-correlation in a set of stocks," Quantitative Finance, vol. 1, 2001.

[5] M. Tumminello, T. Di Matteo, T. Aste, and R. N. Mantegna, "Correlation based networks of equity returns sampled at different time horizons," The European Physical Journal B, vol. 55, pp. 209-217, 2007.

[6] G. F. Zebende, M. F. Da Silva, and A. Machado Filho, "DCCA cross-correlation coefficient differentiation: theoretical and practical approaches," Physica A: Statistical Mechanics and Its Applications, vol. 392, no. 8, pp. 1756-1761, 2013.

[7] C. Hamzacebi and M. Pekkaya, "Determining of stock investments with grey relational analysis," Expert Systems with Applications, vol. 38, pp. 9186-9195, 2011.

[8] G. F. Zebende, "DCCA cross-correlation coefficient: quantifying level of cross-correlation," Physica A: Statistical Mechanics and Its Applications, vol. 390, no. 4, pp. 614-618, 2011.

[9] K. A. Kim and S. G. Rhee, "Price limit performance: evidence from the tokyo stock exchange," The Journal of Finance, vol. 52, no. 2, pp. 885-901, 1997.

[10] X. Xiong, D. Nan, Y. Yang, and Z. Yongjie, "Study on market stability and price limit of Chinese stock index futures market: an agent-based modeling perspective," PLoS One, vol. 10, Article ID e0141605, 2015.

[11] S. H. Chan, K. A. Kim, and S. G. Rhee, "Price limit performance: evidence from transactions data and the limit order book," Journal of Empirical Finance, vol. 12, no. 2, pp. 269-290, 2005.

[12] L. Kristoufek, "Measuring correlations between non-stationary series with DCCA coefficient," Physica A: Statistical Mechanics and Its Applications, vol. 402, pp. 291-298, 2014.

[13] L. Kristoufek, "Detrending moving-average cross-correlation coefficient: measuring cross-correlations between non-stationary series," Physica A: Statistical Mechanics and Its Applications, vol. 406, pp. 169-175, 2014.

[14] Y. Yin and P. Shang, "Modified DFA and DCCA approach for quantifying the multiscale correlation structure of financial markets," Physica A: Statistical Mechanics and Its Applications, vol. 392, no. 24, pp. 6442-6457, 2013.

[15] E. Guedes, A. Dionísio, P. J. Ferreira, and G. F. Zebende, "DCCA cross-correlation in blue-chips companies: a view of the 2008 financial crisis in the Eurozone," Physica A:
Statistical Mechanics and Its Applications, vol. 479, pp. 38-47, 2017.

[16] M. F. Da Silva, É. J. d. A. Leão Pereira, A. M. Da Silva Filho, A. P. Nunes De Castro, J. G. V. Miranda, and G. F. Zebende, "Quantifying cross-correlation between Ibovespa and Brazilian blue-chips: the DCCA approach," Physica A: Statistical Mechanics and Its Applications, vol. 424, pp. 124-129, 2015.

[17] G. F. Zebende, A. A. Brito, and A. P. Castro, "DCCA crosscorrelation analysis in time-series with removed parts," Physica A: Statistical Mechanics and Its Applications, vol. 545, p. 123472, 2020.

[18] L. Piao and Z. Fu, "Quantifying distinct associations on different temporal scales: comparison of DCCA and Pearson methods," Scientific Reports, vol. 6, 2016.

[19] R. T. Vassoler and G. F. Zebende, "DCCA cross-correlation coefficient apply in time series of air temperature and air relative humidity," Physica A: Statistical Mechanics and Its Applications, vol. 391, no. 7, pp. 2438-2443, 2012.

[20] N. Yuan and Z. Fu, "Different spatial cross-correlation patterns of temperature records over China: a DCCA study on different time scales," Physica A: Statistical Mechanics and Its Applications, vol. 400, pp. 71-79, 2014.

[21] Y. Chen, L. Cai, R. Wang et al., "DCCA cross-correlation coefficients reveals the change of both synchronization and oscillation in EEG of Alzheimer disease patients," Physica A: Statistical Mechanics and Its Applications, vol. 490, pp. 171184, 2018.

[22] B. Podobnik and H. E. Stanley, "Detrended cross-correlation analysis: a new method for analyzing two nonstationary time series," Physical Review Letters, vol. 100, 2008.

[23] G.-J. Wang, C. Xie, Y.-J. Chen, and S. Chen, "Statistical properties of the foreign exchange network at different time scales: evidence from detrended cross-correlation coefficient and minimum spanning tree," Entropy, vol. 15, no. 12, pp. 1643-1662, 2013.

[24] S. Li, X. Lu, and X. Liu, "Dynamic relationship between Chinese RMB exchange rate index and market anxiety: a new perspective based on MF-DCCA," Physica A: Statistical Mechanics and Its Applications, vol. 541, p. 123405, 2020.

[25] Q. Ruan, S. Zhang, D. Lv, and X. Lu, "Financial liberalization and stock market cross-correlation: MF-DCCA analysis based on Shanghai-Hong Kong Stock Connect," Physica A: Statistical Mechanics and Its Applications, vol. 491, pp. 779-791, 2018.

[26] X. Sun and Z. Liu, "Optimal portfolio strategy with crosscorrelation matrix composed by DCCA coefficients: evidence from the Chinese stock market," Physica A: Statistical Mechanics and Its Applications, vol. 444, pp. 667-679, 2016.

[27] T. M. Dao, F. McGroarty, and A. Urquhart, "Ultra-highfrequency lead-lag relationship and information arrival," Quantitative Finance, vol. 18, no. 5, pp. 725-735, 2018.

[28] V. Arakelian and S. Qamhieh Hashem, "The leaders, the laggers, and the "vulnerables"," Risks, vol. 8, p. 26, 2020.

[29] K. Hou, "Industry information diffusion and the lead-lag effect in stock returns," The Review of Financial Studies, vol. 20, pp. 1113-1138, 2007.

[30] A. W. Lo and A. C. MacKinlay, "When are contrarian profits due to stock market overreaction?," The Review of Financial Studies, vol. 3, pp. 175-205, 2015.

[31] J.-P. Onnela, K. Kaski, and J. Kertész, "Clustering and information in correlation based financial networks," The European Physical Journal B-Condensed Matter, vol. 38, no. 2, pp. 353-362, 2004. 
[32] M. Ausloos and R. Lambiotte, "Clusters or networks of economies? A macroeconomy study through Gross Domestic Product," Physica A: Statistical Mechanics and Its Applications, vol. 382, no. 1, pp. 16-21, 2007.

[33] S. Kumar and N. Deo, "Correlation and network analysis of global financial indices," Physical Review E, vol. 86, p. 026101, 2012.

[34] H. Li, H. An, W. Fang, Y. Wang, W. Zhong, and L. Yan, "Global energy investment structure from the energy stock market perspective based on a heterogeneous complex network model," Applied Energy, vol. 194, 2017.

[35] P. Gai and S. Kapadia, "Contagion in financial networks," Proceedings of the Royal Society A: Mathematical, Physical and Engineering Sciences, vol. 466, no. 2120, pp. 2401-2423, 2010.

[36] R. Kali and J. Reyes, "Financial contagion on the international trade network," Economic Inquiry, vol. 48, no. 4, pp. 1072-1101, 2010.

[37] L. Zhao, J. Wang, R. Huang, H. Cui, X. Qiu, and X. Wang, "Sentiment contagion in complex networks," Physica A: Statistical Mechanics and Its Applications, vol. 394, pp. 17-23, 2014.

[38] J. Papenbrock and P. Schwendner, "Handling risk-on/risk-off dynamics with correlation regimes and correlation networks," Financial Markets and Portfolio Management, vol. 29, pp. 125-147, 2015.

[39] N. Hautsch, J. Schaumburg, and M. Schienle, "Financial network systemic risk contributions," Review of Finance, vol. 19, no. 2, pp. 685-738, 2015.

[40] M. Billio, M. Getmansky, A. W. Lo, and L. Pelizzon, "Econometric measures of connectedness and systemic risk in the finance and insurance sectors," Journal of Financial Economics, vol. 104, no. 3, pp. 535-559, 2012.

[41] C. Liu and N. Arunkumar, "Risk prediction and evaluation of transnational transmission of financial crisis based on complex network," Cluster Computing, vol. 22, 2019.

[42] D. A. Hsu, "A bayesian robust detection of shift in the risk structure of stock market returns," Journal of the American Statistical Association, vol. 77, no. 377, pp. 29-39, 1982.

[43] U. Ayub, S. Z. A. Shah, and Q. Abbas, "Robust analysis for downside risk in portfolio management for a volatile stock market," Economic Modelling, vol. 44, pp. 86-96, 2015.

[44] M. E. J. Newman, "The structure and function of complex networks," SIAM Review, vol. 45, no. 2, pp. 167-256, 2003.

[45] V. D. Blondel, J. Guillaume, R. Lambiotte, and E. Lefebvre, "Fast unfolding of communities in large networks," Journal of Statistical Mechanics: Theory and Experiment, vol. 2008, 2008.

[46] R. M. May, "Will a large complex system be stable?," Nature, vol. 238, no. 5364, pp. 413-414, 1972.

[47] F. Juhász, The May-Wigner Theorem For Block Random Matrices, Computer and Automation Institute Hungarian Academy of Sciences, Budapest, Hungary, 1994.

[48] Y. V. Fyodorov and B. A. Khoruzhenko, "Nonlinear analogue of the May-Wigner instability transition," Proceedings of the National Academy of Sciences, vol. 113, no. 25, pp. 6827-6832, 2016.

[49] S. A. Levin, G. Sugihara, and R. M. May, "Complex systems Ecology for bankers," Nature, vol. 451, pp. 893-895, 2008.

[50] R. H. Heiberger, "Stock network stability in times of crisis," Physica A: Statistical Mechanics and Its Applications, vol. 393, pp. 376-381, 2014.

[51] A. Namaki, A. H. Shirazi, R. Raei, and G. R. Jafari, "Network analysis of a financial market based on genuine correlation and threshold method," Physica A: Statistical Mechanics and Its Applications, vol. 390, no. 21-22, pp. 3835-3841, 2011.
[52] M. E. J. Newman and D. J. Watts, "Scaling and percolation in the small-world network model," Physical Review E, vol. 60, no. 6, pp. 7332-7342, 1999.

[53] C. Fang and F. Marle, "Dealing with project complexity by matrix-based propagation modelling for project risk analysis," Journal of Engineering Design, vol. 24, no. 4, pp. 239-256, 2013.

[54] M. Amiri, M. Zandieh, B. Vahdani, R. Soltani, and V. Roshanaei, "An integrated eigenvector-DEA-TOPSIS methodology for portfolio risk evaluation in the FOREX spot market," Expert Systems With Applications, vol. 37, no. 1, pp. 509-516, 2010.

[55] P. Bonacich, "Factoring and weighting approaches to status scores and clique identification," The Journal of Mathematical Sociology, vol. 2, no. 1, pp. 113-120, 1972.

[56] J. Hung, Y. Liu, I. Jiang, and S. Liang, "Price discovery and trading activity in Taiwan stock and futures markets," Emerging Markets Finance and Trade, vol. 56, pp. 963-976, 2020.

[57] R. K. Albert, H. Jeong, and A. L. Barabasi, "Error and attack tolerance of complex networks," Nature, vol. 27, 2000.

[58] P. Crucitti, V. Latora, M. Marchiori, and A. Rapisarda, "Error and attack tolerance of complex networks," Nature, vol. 340, pp. 378-382, 2000. 